\title{
DERECHO Y POLÍTICAS AMBIENTALES EN ASTURIAS (SEGUNDO SEMESTRE 2020)
}

\author{
ALEJANDRA BOTO ÁLVAREZ \\ Profesora Titular de Derecho Administrativo \\ Universidad de Oviedo
}


SUMARIO: 1. Introducción: Confinamiento, pandemia y medio ambiente.- 2. Distintas medidas en materia de alimentación ecológica y de calidad.- 3. La nueva organización administrativa del medio ambiente.- 4. Movilidad metropolitana: multimodal y sostenible... algún día.

\section{INTRODUCCIÓN: CONFINAMIENTO, PANDEMIA Y MEDIO AMBIENTE ${ }^{1}$}

Cualquier crónica temática que haya de cubrir los últimos meses ha de hacer referencia a la crisis sanitaria generada por el COVID-19 y sus efectos en el sector en que se trate. Las conexiones entre medio ambiente y sanidad son evidentes, como ya se tuvo ocasión de apuntar en la entrega correspondiente a esta sección hace justo un año (al hilo de la aprobación de la Ley 7/2019, de 29 de marzo, de salud del Principado de Asturias), y se ha vuelto a señalar recientemente por la doctrina 2 .

No puede ser menos esta revisión de las medidas normativas asturianas más recientes con incidencia ambiental, muchas de las cuales como se verá traen causa directa en la situación de confinamiento que se vivió a partir de mediados de marzo, con efectos evidentes sobre consumo y alimentación. La situación ha abierto también un proceso de reflexión sobre la organización y el funcionamiento administrativo, que se ha materializado ya en una remodelación de las Consejerías y en una nueva configuración de las instancias con responsabilidad ambiental. Además, se han producido también novedades en relación con el futuro modelo de movilidad metropolitana.

\section{DISTINTAS MEDIDAS EN MATERIA DE ALIMENTACIÓN ECOLÓGICA Y DE CALIDAD}

Durante los últimos meses han llegado a las páginas del BOPA toda una serie de disposiciones relacionadas con distintivos de calidad en productos alimentarios, en algunos casos como consecuencia directa de la situación sanitaria. Es el caso, sin ir más lejos, de la Resolución de 30 de abril de 2020, de la Consejería de Desarrollo Rural, Agroganadería y Pesca, por la que se

\footnotetext{
${ }^{1}$ El trabajo ha sido elaborado en el marco del proyecto de investigación de referencia IDI/2018/000219 correspondiente a una ayuda a organismos públicos de investigación para apoyar las actividades de grupos de investigación en el Principado de Asturias concedida por el Gobierno de este al grupo SPAG (Servicios Públicos, Administraciones y Garantías) que coordina el profesor Tolivar Alas en la Universidad de Oviedo.

${ }^{2}$ Por todos, puede verse el editorial de Alenza García, J. F., "El Derecho ambiental como vacuna y como vitamina (crónica iusambientalista de la pandemia)", Revista Aranzadi de Derecho Ambiental, núm. 46, 2020.
} 
modifica temporalmente el pliego de condiciones de la indicación geográfica protegida "Ternera Asturiana”, publicada en el BOPA núm. 88 de 8 de mayo.

En efecto, de las medidas dictadas a partir del mes de marzo para contener la pandemia se derivan afecciones económicas no menores y, consecuentemente, también afectan a diferentes ámbitos de la producción y la comercialización agroalimentaria, particularmente en lo que se refiere a la suspensión y limitación de las actividades de hostelería y restauración. Las restricciones afectaron notablemente al sector de la carne de vacuno en general y a la indicación geográfica protegida (IGP) Ternera Asturiana en particular, dado que comercializa parte de su producción, y en especial partes muy concretas de las canales certificadas (sobre todo partes nobles, como chuleteros o solomillos) en establecimientos de la hostelería y la restauración. Por ello, el Consejo Regulador solicitó una modificación temporal del pliego de condiciones de esta indicación geográfica en el sentido de que se permitiese excepcionalmente la congelación de las carnes, práctica en condiciones normales no permitida. El mantenimiento de la certificación de Ternera Asturiana en piezas de carne que los operadores autorizados congelasen durante un período de tiempo delimitado habría de permitir posteriormente su salida equilibrada al mercado, y servir para atenuar las dificultades que las medidas de contención provocaron en la cadena de producción, especialmente en las ganaderías e industrias inscritas en la marca. A ello accedió la Comunidad Autónoma, disponiendo que las piezas de carne amparadas por la protección de la IGP Ternera Asturiana, procedentes de canales certificadas durante la vigencia del estado de alarma y/o medidas similares decretadas en España adoptadas por las autoridades estatales o autonómicas competentes que provoquen restricciones en la comercialización de la carne de vacuno en los establecimientos de hostelería y restauración como consecuencia del COVID-19, podrán someterse a procesos de congelación mientras durasen estas medidas. En todo caso, estas piezas de carne podrán comercializarse bajo la protección de la IGP al consumidor final sólo hasta el 31 de diciembre del año 2020. Además, dichas piezas de carne congeladas deberán cumplir los requisitos aplicables a la comercialización de carne congelada de vacuno, especialmente en lo relativo a la información al consumidor sobre el 
proceso de congelación y la fecha de consumo preferente, así como a la excepcionalidad de su comercialización bajo la protección de la IGP.

Por otro lado, los problemas de comercialización de productos alimentarios como el marisco a consecuencia de las medidas de carácter extraordinario adoptadas con la declaración del estado de alarma para la gestión de la situación de crisis sanitaria ocasionada por el COVID-19 llevó, en marzo, a acordar el cierre de la actividad de extracción del percebe y del marisqueo a pie en general (Resolución de 17 de marzo de 2020, publicada en BOPA núm. 57, de 23 de marzo). La reapertura de la actividad se acordó a comienzos del mes de mayo, al apreciarse un cierto incremento en la demanda de los productos marisqueros, con una relativa recuperación de los canales de comercialización y, por tanto, de obtención de precios relativamente rentables (Resolución de 6 de mayo de 2020, publicada en BOPA núm. 89, de 11 de mayo).

Sigue prohibida sin embargo la pesca, tanto comercial como recreativa, del oricio (Paracentrotus lividus), debido a la situación de la especie. La extracción del recurso lleva vedada en la región desde 2016 y se ha ido prorrogando de manera sucesiva porque los resultados del proceso que la Administración autonómica, en colaboración con la Universidad de Oviedo, ha llevado a cabo para el seguimiento, estudio y evaluación de la especie, con realización de muestreos en distintos puntos del litoral asturiano, junto con labores de repoblación, revelan que no se observa una evolución favorable de las poblaciones, salvo en lugares puntuales del occidente asturiano, y que el recurso en general no presenta un estado que permita en modo alguno una explotación comercial o una extracción recreativa. La veda se estableció inicialmente por un período de dos años (Resolución de 5 de septiembre de 2016, de la Consejería de desarrollo rural y recursos naturales), revisable en función de la evolución apreciada del recurso; se prorrogó en 2018 por otros dos años más (Resoluciones de 7 de septiembre y de 18 de diciembre), y ahora se acuerda que continúe de manera indefinida, hasta que se constate una recuperación adecuada (Resolución de 23 de septiembre de 2020, publicada en BOPA núm. 189, de 29 de septiembre).

Más allá de la concreta situación del marisco y la carne, los distintivos de calidad han protagonizado de manera bastante intensa la actualidad asturiana en los últimos tiempos. Así, en el BOPA núm. 81, de 28 de abril, se publicaba la 
Resolución de 20 de abril de 2020, de la Consejería de desarrollo rural, agroganadería y pesca, modificando el reglamento de uso de la marca "Alimentos del Paraíso Natural" para simplificar el acceso a la misma por quienes producen en el marco de una IGP o de una Denominación de Origen Protegida (DOP).

Estas se están también modernizando y así varios estatutos de Consejos Reguladores vieron la luz, por cierto, durante el mes de agosto (Resoluciones de 31 de julio de 2020, de la Consejería de Medio Rural y Cohesión Territorial, en BOPA núm. 154, de 10 de agosto y núm. 155, del día siguiente): DOP de los quesos "Cabrales", "Casin", "Gamonéu" y "Afuega'l Pitu", de la "Sidra de Asturias" y del vino de "Cangas"; e IGP "Chosco de Tineo", "Faba Asturiana" y "Ternera Asturiana".

El salto en pro de la calidad y la seguridad alimentarias también se puede apreciar en la Resolución de 30 de abril de 2020, de la Consejería de Desarrollo Rural, Agroganadería y Pesca, por la que se aprueban los estatutos del Consejo de la Producción Agraria Ecológica del Principado de Asturias (BOPA núm. 88 de 8 de mayo) y en la subsiguiente Resolución de la misma Consejería de 13 de mayo aprobando convocatoria de subvenciones para el fomento de la comercialización de alimentos ecológicos en el Principado de Asturias en 2020 (extracto en BOPA núm. 99, de 25 de mayo).

La regulación original del Consejo de la Producción Agraria Ecológica del Principado de Asturias estaba contenida en el Decreto 81/2004 de 21 de octubre, sobre la producción agraria ecológica y su indicación en los productos agrarios y alimenticios. Se hacía necesario aprobar unos nuevos estatutos del mismo de conformidad con lo previsto en la Ley del Principado de Asturias 2/2019, de 1 de marzo, de calidad alimentaria, calidad diferenciada y venta directa de productos alimentarios, que aún no había habido ocasión de traer a esta sección. Esta Ley, que contempla entre sus fines el fomento de una producción agraria y agroalimentaria, así como de los productos pesqueros, con calidad diferenciada, equidad social y sostenibilidad ambiental configura, en su artículo 36 , la entidad de gestión de la producción ecológica como corporación de derecho público con personalidad jurídica propia y plena capacidad de obrar. 
El Consejo de la Producción Agraria Ecológica del Principado de Asturias se establece como única autoridad de control en materia de producción ecológica en el Principado de Asturias, encargada de la aplicación del sistema de control establecido en la normativa europea sobre producción y etiquetado de los productos ecológicos. Tiene conferidas las facultades de inspección y de certificación en el ámbito de la producción ecológica de conformidad con las disposiciones previstas en la normativa vigente en la materia, además de la representación, la defensa, la garantía y la promoción de la producción ecológica y de los productos por ella amparados. Está formado por todos los operadores que participen en cualquier etapa de la producción, la preparación, la distribución y la comercialización relativas a los productos ecológicos que figuren debidamente certificados y se encuentren al corriente de sus obligaciones económicas para con el Consejo.

El sistema reposa entonces sobre un Registro de operadores ecológicos de Asturias, como registro de carácter administrativo de la Consejería competente en materia de producción ecológica que gestiona y actualiza el Consejo y en el que deben inscribirse todos los operadores de producción ecológica del Principado sometidos al régimen de control. Este Registro autonómico se estructura en consonancia con el Registro General de Operadores Ecológicos (REGOE), con el fin de garantizar la interoperabilidad entre ambos. Los estatutos desarrollan además los órganos de gobierno del Consejo (Pleno y presidencia) y los órganos ejecutivos (dirección técnica, Comité de Certificación y Comité de Partes). En el Pleno están representados los productores agrícolas y ganaderos, los elaboradores y los comercializadores, ya sean distribuidores, mayoristas 0 importadores, y los comercios minoristas. También existen representantes de la Administración y de los consumidores, que podrán asistir con voz, pero sin voto. El Consejo de la Producción Agraria Ecológica del Principado de Asturias, en su condición de corporación de derecho público y en el ámbito del ejercicio de sus funciones públicas, actúa de acuerdo con el Derecho Administrativo y ejerce las potestades inherentes a la Administración Pública. Los Estatutos disponen que las decisiones, los acuerdos y los actos del Consejo sujetos a Derecho Administrativo ponen fin a la vía administrativa y pueden ser objeto de recurso directamente ante la jurisdicción contenciosa administrativa por las personas 
afectadas, o bien recurso potestativo de reposición ante el órgano que los dictó. Por su parte, respecto a los acuerdos y los actos dictados en ejercicio de funciones delegadas por la Administración, se dispone la procedencia de un recurso de alzada (impropio) ante la Administración delegante.

\section{LA NUEVA ORGANIZACIÓN ADMINISTRATIVA DEL MEDIO AMBIENTE}

La excepcional coyuntura sociosanitaria ha cambiado por completo el marco en el que se aprobó el Decreto 13/2019, de 24 de julio, del Presidente del Principado de Asturias, de reestructuración de las Consejerías que integran la Administración de la Comunidad Autónoma³.

El nuevo contexto nos sitúa ante nuevos retos en el ámbito de la organización de la Administración, que requiere intensificar los principios de agilidad, eficacia y eficiencia, articulando así una administración autonómica propia del siglo XXI. Por otro lado, la crisis del coronavirus ha reforzado la necesidad de trabajar por la cohesión territorial, incorporando la perspectiva del desarrollo rural en la de ordenación del territorio, la construcción de infraestructuras y la articulación de la red de transportes.

La constatación de lo anterior llevó a la aprobación del Decreto 6/2020, de 23 de junio, del Presidente del Principado, de segunda modificación parcial del Decreto 13/2019, publicado en el BOPA núm. 121, de 24 de junio. Ese Decreto renombra la Consejería de Infraestructuras, Medio Ambiente y Cambio Climático como Consejería de Administración Autonómica, Medio Ambiente y Cambio Climático, y la Consejería de Desarrollo Rural, Agroganadería y Pesca como Consejería de Medio Rural y Cohesión Territorial. La reforma pretende subrayar las competencias de la primera Consejería para una reforma de la función pública autonómica, mientras que las cuestiones de infraestructuras pasan a la segunda, que se ve así reforzada en la medida en que la epidemia ha aumentado la importancia del medio rural, para el que la necesidad de infraestructuras resulta

\footnotetext{
${ }^{3}$ Comentado en la entrega correspondiente al vol X, núm. 2 (2019).
} 
esencial, para la cohesión del territorio y aprovechar mejor el potencial de las zonas rurales.

La nueva estructura de la Consejería de Administración Autonómica, Medio Ambiente y Cambio Climático se publicaría el 3 de julio siguiente, en el Decreto 33/2020, de 2 de julio (BOPA núm. 128). En esa misma fecha se publicó también el Decreto 39/2020, por el que se establece la estructura orgánica básica de la Consejería de Medio Rural y Cohesión Territorial, que queda como el órgano superior de la Administración del Principado de Asturias al que le corresponde la propuesta y ejecución de la política del Gobierno en materia de medio natural, de recursos agrícolas, forestales, ganaderos y pesqueros, de industria agroalimentaria y de desarrollo rural, así como de infraestructuras viarias y portuarias, transportes, conectividad y movilidad, ordenación del territorio y urbanismo.

El Medio Ambiente sigue presente en la denominación formal de la Consejería de Administración Autonómica, que también se identifica como la competente en Cambio Climático, pero es indiscutible que la Consejería de Medio Rural ve reforzado su rol de liderazgo en la materia.

\section{MOVILIDAD METROPOLITANA: MULTIMODAL Y SOSTENIBLE... ALGÚN} DÍA

Una vez más volvemos a ocuparnos del modelo de movilidad metropolitana en la región central de Asturias, en relación en este caso con el borrador del Plan de movilidad multimodal en el Área metropolitana del Principado de Asturias, que junto con su estudio ambiental estratégico se sometía a información pública en marzo de este año, tal y como se relató en la crónica correspondiente al número precedente de esta revista.

Contenía diferentes propuestas para fomentar los modos de transporte no motorizados, incrementar la movilidad en transporte público por carretera, impulsar el uso del ferrocarril y racionalizar el uso del vehículo privado. Entre ellas, destacaba la creación de ejes cívicos entre los centros urbanos y la periferia, carriles reservados al transporte público en la entrada a las ciudades, y aparcamientos disuasorios. 
El último movimiento en relación con ello ha sido la publicación, en el BOPA núm. 140, de 21 de julio, del anuncio de suspensión de ese trámite de información pública. La razón que se esgrime por parte de la Consejería de medio rural y cohesión territorial es la necesidad de coordinación de ese instrumento con la Estrategia de movilidad sostenible del Principado de Asturias, prevista por el artículo 14 de la Ley del Principado de Asturias 12/2018, de 23 de noviembre, de Transportes y Movilidad Sostenible, y que también se estaría tramitando en estos momentos.

La aspiración de coordinación debe sin duda saludarse. El nuevo retraso en el diseño efectivo de la movilidad del futuro en la zona central de Asturias, sin embargo, es una muestra más de las trabas y dificultades que rodean la cuestión, controvertida desde su origen, que puede remontarse a las Directrices subregionales del área central de marzo de 2016, y que la aprobación de la Ley 12/2018 no parece haber salvado, por lo menos de momento, a pesar de que hayan transcurrido ya casi dos años desde su entrada en vigor $Y$ ello a pesar de que la Ley expresamente considerase el Área Metropolitana Central de Asturias como un ámbito de actuación concreto, en particular en sus artículos 8, 9 y 16; tal y como ya se comentara con ocasión de la crónica sobre la Ley en la entrega de esta sección correspondiente al vol. X, núm. 1 (2019).

En efecto, el artículo 8 de la Ley 12/2018 identifica en la zona un interés metropolitano que exige la coordinación y cooperación integradas del transporte público, tanto entre la fase urbana e interurbana de los viajes como entre los diferentes modos de transporte. Se recuerda expresamente que la colaboración administrativa en este ámbito territorial ha de tener como finalidad la garantía de la intermodalidad y del mejor servicio en rapidez, frecuencia, calidad y comodidad, con el menor coste económico, social, energético y medioambiental, así como la mayor racionalidad en el uso del suelo y la ordenación territorial. Los servicios e infraestructuras de transporte público de interés metropolitano en el Área Metropolitana Central de Asturias se definen en el artículo 9. Por su parte, el artículo 16, identifica con ese Área el ámbito de uno de los Planes Supramunicipales de Movilidad Sostenible que habrán de aprobarse en desarrollo de la misma Ley y de la Estrategia de movilidad sostenible del Principado de Asturias. Puede verse una relación directa con el instrumento que 
estaba en información pública, si bien huelga recordar que el Área Metropolitana no existe como entidad administrativa y que los perfiles de los distintos instrumentos, comenzados a elaborar en momentos y contextos diferentes, y sin lograr todavía pasar de la fase de borrador, se dan todos en clave de soft law.

Que la Estrategia de Movilidad Sostenible del Principado de Asturias resulta vinculante para el resto de instrumentos de planificación de la movilidad en el Principado de Asturias es algo que no cabe discutir. El tiempo pasa, no obstante, y la sensación es de nulo avance. Sólo cabe ahora seguir atentos a la evolución en la tramitación entonces de la Estrategia y de sus instrumentos de desarrollo, de lo que esperamos dar cuenta de manera próxima en esta revista. 